\title{
CLOSED FORM EXPRESSIONS FOR INTEGRAL RAY GEOMETRIC PARAMETERS FOR WAVE PROPAGATION ON GENERAL QUADRIC SURFACES OF REVOLUTION
}

\author{
RM Jha, SA Bokhari, V Sudhakar and PR Mahapatra \\ Department of Aerospace Engineering \\ Indian Institute of Science \\ Bangalore, 560012 India
}

Summary

The quadric surfaces of revolution (QUASORs) consisting of right circular cone, sphere, ellipsoid of revolution and general paraboloid and hyperboloid of revolution, which are coordinate surfaces of the Eisenhart coordinate systems, form a versatile class of surfaces from the surface modeling point of view. (The right circular cylinder has been treated elsewhere by the authors as a quadric cylinder). The Eisenhart coordinate system permits a simple separation of the scalar and vector potentials in the field equations.

The ray-theoretic formulation developed by Pathak et al [1] for the determination of the radiation characteristics of the antennas in the vicinity of azimuth-symmetric quadrics is hindered by the fact that the integral surface ray geometric parameters are not readily available. This difficulty mainly arises due to the fact that, unlike the quadric cylinders, the QUASORs in general are non-developable.

We summarily present a table of these ray parameters consisting of (i) the relation between the geodesic coordinates $v$ and $u$,

(ii) the arc length

and (iii) the generalized Fock parameter for the complete class of the QUASORs.

A Geodesic Constant Method (GCM) developed by the authors permits the derivation of these ray parameters in terms of the geodesic constant $h$ alone. since $h$ can be expressed in terms of the source and observation point coordinates in the case of sphere and cone, in these cases the ray parameters are in the closed form. On the other hand, in the case of the ellipsoid of revolution and the general paraboloid and hyperboloid of revolution (GPOR and GHOR), h can be obtained using a simple univariate search. Hence in these cases, the ray parameters are in a one-parameter dependent form [2]. Using this approach, it is possible to readily calculate the various radiation characteristics of the antenna in vicinity of a general QUASOR.

\section{REFERENCES}

[1] P.H. Pathak and N. Wang, "Ray analysis of mutual coupling between antennas on a convex surface", IEEE Trans. Antennas \& Propagat. (USA), vol. AP-29, no. 6, pp. 911-922, Nov. 1981.

[2] R.M. Tha, Surface Ray Tracing on Convex Quadrics with Applications to Mutual Coupling between Antennas on Aerospace Bodies, Ph D Dissertation. Submitted to Department of Aerospace Engineering, Indian Institute of Science, Bangalore, India, Nov. 1988. 
TABLE 1

IKTRGRAL SURFACE RAY GOAETRIC PARAMETERS OBTAINED

FOR QUADRIC SURFACES OF REVOLUTION

1. Relation betwen the geodesic coordinates

$\mathrm{u}$ and $\mathrm{v}$ on the geodesic

$v=v(u, h)+h$

Geodesic. coordinates correspondence in individual Eisenhart Coordinates

General Paraboloid of Revolution

$v=\frac{h_{r m}}{a^{2}} \ln \frac{a\left[4 u^{2}+a^{2}\right]^{1 / 2}+2\left[a^{2} u^{2}-h_{r m}^{2}\right]^{1 / 2}}{a\left[4 u^{2}+a^{2}\right]^{1 / 2}-2\left[a^{2} u^{2}-h_{r m}^{2}\right]^{1 / 2}}+\sin ^{-1}\left[\frac{a\left[a^{2} u^{2}-h_{r m}^{2}\right]^{1 / 2}}{u\left[a^{4}+4 h_{r m}^{2}\right]^{1 / 2}}\right]+h_{r m}^{-}$

$\mathrm{u}=\mathrm{u}, \quad \mathrm{v}=\emptyset$

$v=\frac{1}{\sin a} \sec ^{-1}\left[\frac{\mathrm{u} \sin a}{h_{\mathrm{rm}}}\right]+\mathrm{h}_{\mathrm{rm}}^{-}$

$\mathrm{u}=\mathrm{r}, \mathrm{v}=\emptyset$

ััฒ

$v=-\tan ^{-1}\left[\frac{h_{r m} \cos u}{\left(a^{2} \sin ^{2} u-h_{\mathrm{rm}}^{2}\right)^{1 / 2}}\right]+h_{r m}^{-}$

$\mathrm{u}=\theta, \quad \mathrm{v}=\emptyset$

Ellipsoid of Revolution

As a function of incomplete elliptic integrals of first and second kind $\mathrm{E}(\mathrm{u}, \mathrm{k})$ and $\mathrm{F}(\mathrm{u}, \mathrm{k})$, and $\mathrm{h}_{\mathrm{rm}}$ and $\mathrm{h}_{\mathrm{rm}}$
where the modulus $\mathrm{k}$ is a function of shaping parameters $(\mathrm{a}, \mathrm{c})$.

$u=\theta, \quad v=\varnothing$

Ceneral Hyperboloid

$\mathrm{u}=\mathrm{n}, \quad \mathrm{v}=\phi$

As a function of incomplete elliptic integrals of first and second kind $\mathrm{E}(\mathrm{u}, \mathrm{k})$ and $\mathrm{F}(\mathrm{u}, \mathrm{k})$, and $h_{\mathrm{rm}}$ and $h_{\mathrm{rm}}$
where the modulus $\mathrm{k}$ is a function of shaping parameters $(\mathrm{a}, \mathrm{c})$. 
2. Arc length $s=s(u)$

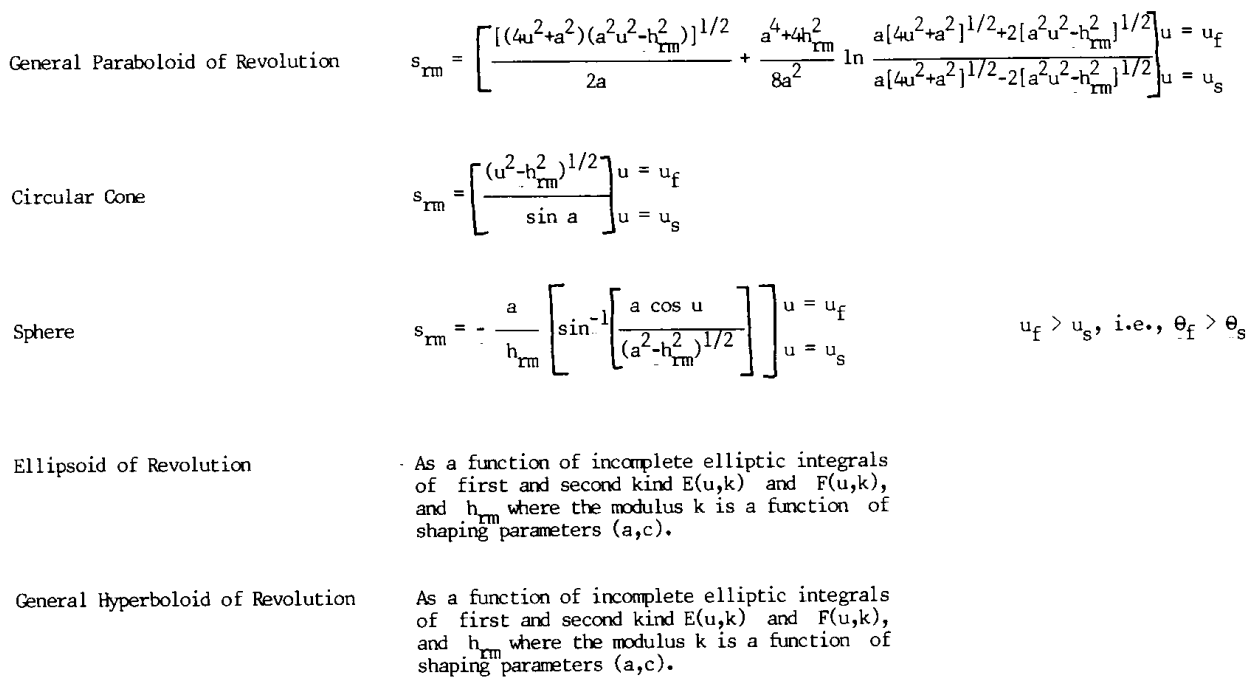




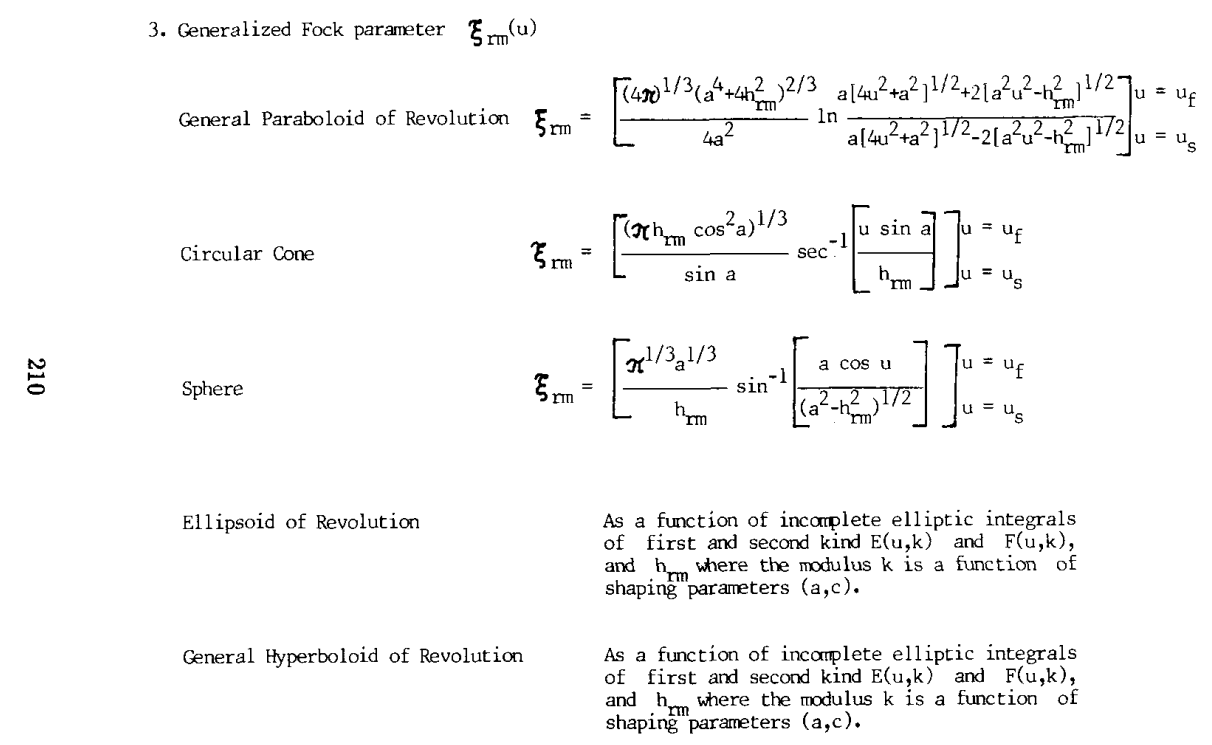

